\title{
On polynomial approximations to solutions of implicit differental equations
}

\author{
Ihor Korol
}




\title{
ON POLYNOMIAL APPROXIMATIONS TO SOLUTIONS OF IMPLICIT DIFFERENTIAL EQUATIONS
}

\author{
IHOR KOROL \\ Mathematical Faculty, Uzhgorod National University \\ Pidhirna 49, 88000 Uzhgorod, Ukraine \\ math1@univ. uzhgorod.ua
}

[Received: April 4, 2002]

\begin{abstract}
In this paper the possibility to present by a polynomial an independent variable for the approximate solutions of the systems of implicit ordinary differential equations under multi-point boundary conditions is substantiated.
\end{abstract}

Mathematical Subject Classification: 34A09, 34B10

Keywords: multi-point boundary value problems, implicit differential equations, numericalanalytic method, determining equation.

\section{Introduction}

There is a large number of methods which mathematicians elaborated for studying boundary value problems (BVPs). In the papers [1], [2] the numerical-analytic method based upon successive approximations was introduced. The polynomial version of this method in which the successive approximations are polynomials was proposed in [1] and then developed in [3], [4] for three- and multi-point boundary conditions. In this paper the issue of existence and approximate construction of the solutions of multipoint boundary conditions for the systems of implicit ordinary differential equations of the first order are studied by using polynomial approximations.

\section{Construction of successive polynomial approximations}

Let us consider a system of implicit equations

$$
\frac{d x}{d t}=f\left(t, x, \frac{d x}{d t}\right)
$$

with a multi-point boundary conditions

$$
A_{0} x(0)+\sum_{k=1}^{q} A_{k} x\left(t_{k}\right)+A_{q+1} x(T)=d,
$$

where $x, d \in R^{n}, \quad f:[0, T] \times D_{1} \times D_{2} \rightarrow R^{n}, D_{1}, D_{2}$ are closed bounded domains in $R^{n}, 0=t_{0}<t_{1}<\ldots<t_{q}<t_{q+1}=T, A_{k}(k=0,1, \ldots, q+1)$ - are $n \times n$ matrices 
so that $\operatorname{det}\left[\sum_{k=1}^{q=1} A_{k} t_{k}\right] \neq 0$.

First of all we will introduce some notations [1].

It is known that for $f(t) \in C[0, T]$ there is a unique polynomial $P_{m}^{0}(t)$ among all the polynomials $P_{m}(t)$ with no more than $m$ degree which is the best approximation for $f(t)$ :

$$
E_{m}(f) \equiv\left\|f(t)-P_{m}^{0}(t)\right\|=\inf _{P_{m}(t)}\left\|f(t)-P_{m}(t)\right\| .
$$

Let us set in the interval $[0, T]$ the nodes

$$
\tau_{i}=\frac{T}{2}\left(\cos \frac{(2 i-1) \pi}{2(p+1)}+1\right), \quad i=1,2, \ldots, p+1,
$$

which are obtained by the substitution $\tau=\frac{T}{2}\left(\tau^{\prime}+1\right)$ from the corresponding zeroes $\tau_{i}^{\prime} \in[-1,1]$ of the Chebyshev polynomials

$$
T_{p+1}(t)=\cos ((p+1) \arccos t) .
$$

For arbitrary continuous function $x_{r}(t)$ by $f^{p}\left(t, x_{r}(t), y_{r}(t)\right)$ we denote the Lagrange interpolation polynomial with $p$ degree and with respect to the points (2.3):

$$
f^{p}\left(t, x_{r}\left(t, x_{0}\right), y_{r}\left(t, x_{0}\right)\right)=\left(f_{1}^{p}\left(t, x_{r}\left(t, x_{0}\right), y_{r}\left(t, x_{0}\right)\right), \ldots, f_{n}^{p}\left(t, x_{r}\left(t, x_{0}\right), y_{r}\left(t, x_{0}\right)\right)\right),
$$

where $y_{r}(t):=\frac{d x_{r}(t)}{d t}, \quad f_{j}^{p}\left(t, x_{r}\left(t, x_{0}\right), y_{r}\left(t, x_{0}\right)\right)=a_{0 j}^{r}+a_{1 j}^{r} t+\ldots+a_{p j}^{r}, \quad j=$

$1,2, \ldots, n, \quad f_{j}^{p}\left(\tau_{i}, x_{r}\left(\tau_{i}\right), y_{r}\left(\tau_{i}\right)\right)=f_{j}\left(\tau_{i}, x_{r}\left(\tau_{i}\right), y_{r}\left(\tau_{i}\right), \quad i=1,2, \ldots, p+1\right.$.

Let us denote by

$$
\begin{gathered}
\overline{\mathcal{L}}\left(f, x, y, t, x_{0}\right)=f\left(t, x\left(t, x_{0}\right), y\left(t, x_{0}\right)\right)-\frac{1}{T} \int_{0}^{T} f\left(s, x\left(s, x_{0}\right), y\left(s, x_{0}\right)\right) d s, \\
\mathcal{L}\left(f, x, y, t, x_{0}\right)=\int_{0}^{t}\left(f\left(\tau, x\left(\tau, x_{0}\right), y\left(\tau, x_{0}\right)\right)-\frac{1}{T} \int_{0}^{T} f\left(s, x\left(s, x_{0}\right), y\left(s, x_{0}\right)\right) d s\right) d \tau .
\end{gathered}
$$

We assume that the following conditions hold for the BVP (2.1), (2.2):

a) the vector-function $f(t, x, y)$ is continuous in $\Omega=[0, T] \times D_{1} \times D_{2}$ (and therefore it is bounded by some vector $M$ ) and Lipschitzian in $x$ and $y$, i.e.,

$\left.\left.|f(t, x, y)| \leq M,|f(t, x, y)-f(t, \bar{x}, \bar{y})| \leq K_{1} \mid x-\bar{x}\right)\left|+K_{2}\right| y-\bar{y}\right) \mid$,

where $M$ and $n \times n$ matrices $K_{1}, K_{2}$ have non-negative components. The absolute value sign and the inequalities we understand component-wise;

b) domains $D_{1}$ and $D_{2}$ satisfy the conditions

$$
D_{\beta_{1}}:=\left\{x \in R^{n} \mid B\left(x, \beta_{1}(x)\right) \subset D_{1}\right\} \neq \varnothing, \quad B\left(0, \beta_{2}(x)\right) \subset D_{2},
$$


where $B(x, \rho(x))$ is the ball of radius $\rho(x)$ with center $x$ and

$$
\begin{gathered}
\beta_{1}(x)=\left(\frac{T}{2} E+G\right) \cdot\left(M^{\prime}+L_{p}\right)+T|d(x)|, \quad G=T \cdot \sum_{k=1}^{q}\left|H A_{k}\right| \cdot \alpha_{1}\left(t_{k}\right), \\
\beta_{2}(x)=2\left(M+L_{p}\right)+\frac{1}{T} G\left(M^{\prime}+L_{p}\right)+|d(x)|, \quad H=\left[\sum_{k=1}^{q+1} A_{k} t_{k}\right]^{-1}, \\
d(x)=H \cdot\left(d-\sum_{k=0}^{q+1} A_{k} x\right), \quad \alpha_{1}(t)=2 t\left(1-\frac{t}{T}\right), \\
M^{\prime}=\frac{1}{2}\left[\max _{(t, x, y) \in \Omega} f(t, x, y)-\min _{(t, x, y) \in \Omega} f(t, x, y)\right] \\
L_{p}=(5+\lg p) \max _{r} E_{p}\left(f\left(t, x_{r}^{p+1}\left(t, x_{0}\right), y_{r}^{p}\left(t, x_{0}\right)\right)\right)= \\
=(5+\lg p) \cdot\left(\max _{r} E_{p}\left(f_{1}\left(t, x_{r}^{p+1}\left(t, x_{0}\right), y_{r}^{p}\left(t, x_{0}\right)\right)\right), \ldots\right. \\
\left.\ldots, \max _{r} E_{p}\left(f_{n}\left(t, x_{r}^{p+1}\left(t, x_{0}\right), y_{r}^{p}\left(t, x_{0}\right)\right)\right)\right)
\end{gathered}
$$

c) the eigenvalues $\lambda_{j}(Q)$ of the matrix $Q=K_{1}\left(\frac{T}{2} E+G\right)+K_{2}\left(2 E+\frac{1}{T} G\right)$ satisfy the inequalities

$$
\left|\lambda_{j}(q)\right|<1, \quad j=1, \ldots, n .
$$

Let us introduce the sequence of polynomials with $p+1$ degree

$$
\begin{gathered}
x_{m}^{p+1}\left(t, x_{0}\right)=x_{0}+\mathcal{L}\left(f^{p}, x_{m-1}^{p+1}, y_{m-1}^{p}, t, x_{0}\right)+t H d\left(x_{0}\right)- \\
-t H \sum_{k=1}^{q} A_{k} \mathcal{L}\left(f^{p}, x_{m-1}^{p+1}, y_{m-1}^{p}, t_{k}, x_{0}\right), x_{0}^{p+1}\left(t, x_{0}\right)=x_{0}, m=1,2, \ldots
\end{gathered}
$$

Their derivatives look as follows:

$$
\begin{gathered}
y_{m}^{p}\left(t, x_{0}\right)=\overline{\mathcal{L}}\left(f^{p}, x_{m-1}^{p+1}, y_{m-1}^{p}, t, x_{0}\right)+H d\left(x_{0}\right)- \\
-H \sum_{k=1}^{q} A_{k} \mathcal{L}\left(f^{p}, x_{m-1}^{p+1}, y_{m-1}^{p}, t_{k}, x_{0}\right), y_{0}^{p}\left(t, x_{0}\right)=0, m=1,2, \ldots
\end{gathered}
$$

Here the above index means that this expression is a polynomials of a correspondent degree. It is easy to see that all the members of the sequence (2.6) satisfy the boundary condition (2.2) for arbitrary $x_{0} \in D_{\beta_{1}}$.

The next theorem establishes the convergence of the sequence (2.6) and the properties of the limit functions.

Theorem 1. Let BVP (2.1), (2.2) satisfy the conditions a)-c). Then:

(1) the sequences (2.6) and (2.7) converge to the functions $x^{*}\left(t, x_{0}\right)$ and $y^{*}\left(t, x_{0}\right)$, respectively, as $m \rightarrow \infty$, uniformly in $\left(t, x_{0}\right) \in[0, T] \times D_{\beta_{1}}$ :

$$
x^{*}\left(t, x_{0}\right)=\lim _{m \rightarrow \infty} x_{m}^{p+1}\left(t, x_{0}\right), \quad y^{*}\left(t, x_{0}\right)=\lim _{m \rightarrow \infty} y_{m}^{p}\left(t, x_{0}\right),
$$


where $y^{*}\left(t, x_{0}\right)=\frac{d x^{*}\left(t, x_{0}\right)}{d t} ;$

(2) the limit function $x^{*}\left(t, x_{0}\right)$ satisfies the "perturbed" BVP

$$
\left\{\begin{array}{c}
\frac{d x}{d t}=f\left(t, x, \frac{d x}{d t}\right)+\Delta\left(x_{0}\right), \\
A_{0} x(0)+\sum_{k=1}^{q} A_{k} x\left(t_{k}\right)+A_{q+1} x(T)=d,
\end{array}\right.
$$

where

$$
\begin{gathered}
\Delta\left(x_{0}\right)=-\frac{1}{T} \int_{0}^{T} f^{p}\left(s, x^{*}\left(s, x_{0}\right), y^{*}\left(s, x_{0}\right)\right) d s+H d\left(x_{0}\right)- \\
-H \sum_{k=1}^{q} A_{k} \mathcal{L}\left(f^{p}, x^{*}, y^{*}, t_{k}, x_{0}\right),
\end{gathered}
$$

with the initial value $x^{*}\left(0, x_{0}\right)=x_{0}$;

(3) the following error estimations hold:

$$
\begin{aligned}
\left|x^{*}\left(t, x_{0}\right)-x_{m}^{p+1}\left(t, x_{0}\right)\right| & \leq\left(\alpha_{1}(t) E+G\right) \cdot W_{m-1}^{p}, \\
\left|y^{*}\left(t, x_{0}\right)-y_{m}^{p}\left(t, x_{0}\right)\right| & \leq\left(2 E+\frac{1}{T} G\right) \cdot W_{m-1}^{p},
\end{aligned}
$$

where

$$
\begin{gathered}
W_{m-1}^{p}=\left[\sum_{i=0}^{m-1} Q^{i}\right] \cdot L_{p}+Q^{m-1}(E-Q)^{-1} . \\
\cdot\left[K_{1}\left\{\left(\frac{T}{2} E+G\right) M^{\prime}+T\left|d\left(x_{0}\right)\right|\right\}+K_{2}\left\{2 M+\frac{1}{T} G M^{\prime}+\left|d\left(x_{0}\right)\right|\right\}\right] .
\end{gathered}
$$

Proof. In addition to (2.6), (2.7) let us introduce the sequence of functions.

$$
\begin{gathered}
x_{m}\left(t, x_{0}\right)=x_{0}+\mathcal{L}\left(f, x_{m-1}, y_{m-1}, t, x_{0}\right)+t H d\left(x_{0)}-\right. \\
-t H \sum_{k=1}^{q} A_{k} \mathcal{L}\left(f, x_{m-1}, y_{m-1}, t_{k}, x_{0}\right), \quad x_{0}\left(t, x_{0}\right)=x_{0}, \quad m=1,2, \ldots, \\
y_{m}\left(t, x_{0}\right):=\frac{d x_{m}\left(t, x_{0}\right)}{d t}=\overline{\mathcal{L}}\left(f, x_{m-1}, y_{m-1}, t, x_{0}\right)+H d\left(x_{0}\right)- \\
-H \sum_{k=1}^{q} A_{k} \mathcal{L}\left(f, x_{m-1}, y_{m-1}, t_{k}, x_{0}\right), \quad y_{0}\left(t, x_{0}\right)=0, \quad m=1,2, \ldots
\end{gathered}
$$

Also we introduce some notations:

$$
\begin{aligned}
x_{m} & :=x_{m}\left(t, x_{0}\right), x_{m}^{p+1}:=x_{m}^{p+1}\left(t, x_{0}\right), r_{m+1}\left(t, x_{0}\right):=\left|x_{m+1}\left(t, x_{0}\right)-x_{m}\left(t, x_{0}\right)\right|, \\
y_{m} & :=y_{m}\left(t, x_{0}\right), y_{m}^{p}:=y_{m}^{p}\left(t, x_{0}\right), \widehat{r}_{m+1}\left(t, x_{0}\right):=\left|y_{m+1}\left(t, x_{0}\right)-y_{m}\left(t, x_{0}\right)\right| .
\end{aligned}
$$

We note [1] that

$$
\left|f^{p}\left(t, x_{m}^{p+1}, y_{m}^{p}\right)-f\left(t, x_{m}^{p+1}, y_{m}^{p}\right)\right| \leq L_{p},
$$

and making use of (2.4) we get

$$
\begin{gathered}
\quad\left|f^{p}\left(t, x_{m}^{p+1}, y_{m}^{p}\right)-f\left(t, x_{m}, y_{m}\right)\right| \leq\left|f^{p}\left(t, x_{m}^{p+1}, y_{m}^{p}\right)-f\left(t, x_{m}^{p+1}, y_{m}^{p}\right)\right|+ \\
+\left|f\left(t, x_{m}^{p+1}, y_{m}^{p}\right)-f\left(t, x_{m}, y_{m}\right)\right| \leq L_{p}+K_{1}\left|x_{m}^{p+1}-x_{m}\right|+K_{2}\left|y_{m}^{p}-y_{m}\right| .
\end{gathered}
$$


Using Lemma 3 of [5] we have that

$$
\begin{gathered}
\left|\mathcal{L}\left(f, x, y, t, x_{0}\right)\right| \leq \alpha_{1}(t) M^{\prime} \leq \frac{T}{2} M^{\prime}, \\
\left|T H \sum_{k=1}^{q} A_{k} \mathcal{L}\left(f, x, y, t_{k}, x_{0}\right)\right| \leq G M^{\prime}, \\
\left|\mathcal{L}\left(f^{p}, x_{m}^{p+1}, y_{m}^{p}, t, x_{0}\right)-\mathcal{L}\left(f, x_{m}^{p+1}, y_{m}^{p}, t, x_{0}\right)\right| \leq \alpha_{1}(t) L_{p}, \\
\left|T H \sum_{k=1}^{q} A_{k}\left[\mathcal{L}\left(f^{p}, x_{m}^{p+1}, y_{m}^{p}, t_{k}, x_{0}\right)-\mathcal{L}\left(f, x_{m}^{p+1}, y_{m}^{p}, t_{k}, x_{0}\right)\right]\right| \leq G L_{p} .
\end{gathered}
$$

We have to show that (2.6) is a Cauchy sequence in the space of continuous vector functions. To begin with, we establish for arbitrary $\left(t, x_{0}\right) \in[0, T] \times D_{\beta_{1}}$, and $m=0,1,2, \ldots$ that $x_{m}^{p+1}\left(t, x_{0}\right) \in D_{1}$ and $y_{m}^{p}\left(t, x_{0}\right) \in D_{2}$ by using $(2.16)-(2.19)$ :

$$
\begin{aligned}
& \left|x_{1}^{p+1}-x_{0}\right| \leq\left|\mathcal{L}\left(f^{p}, x_{0}^{p+1}, y_{0}^{p}, t, x_{0}\right)\right|+\left|T H \sum_{k=1}^{q} A_{k} \mathcal{L}\left(f^{p}, x_{0}^{p+1}, y_{0}^{p}, t_{k}, x_{0}\right)\right|+ \\
& +T\left|d\left(x_{0}\right)\right| \leq\left|\mathcal{L}\left(f^{p}, x_{0}^{p+1}, y_{0}^{p}, t, x_{0}\right)-\mathcal{L}\left(f, x_{0}, y_{0}, t, x_{0}\right)\right|+\left|\mathcal{L}\left(f, x_{0}, y_{0}, t, x_{0}\right)\right|+ \\
& +T\left|d\left(x_{0}\right)\right|+\left|T H \sum_{k=1}^{q} A_{k}\left[\mathcal{L}\left(f^{p}, x_{0}^{p+1}, y_{0}^{p}, t_{k}, x_{0}\right)-\mathcal{L}\left(f, x_{0}, y_{0}, t_{k}, x_{0}\right)\right]\right|+ \\
& +\left|T H \sum_{k=1}^{q} A_{k} \mathcal{L}\left(f, x_{0}, y_{0}, t_{k}, x_{0}\right)\right| \leq\left(\alpha_{1}(t) E+G\right)\left(L_{p}+M^{\prime}\right)+T\left|d\left(x_{0}\right)\right| \leq \beta_{1}\left(x_{0}\right), \\
& +T\left|d\left(x_{0}\right)\right| \leq\left|\mathcal{L}\left(f^{p}, x_{0}^{p+1}, y_{0}^{p}, t, x_{0}\right)-\mathcal{L}\left(f, x_{0}, y_{0}, t, x_{0}\right)\right|+\left|\mathcal{L}\left(f, x_{0}, y_{0}, t, x_{0}\right)\right|+ \\
& +\left|T H \sum_{k=1}^{q} A_{k} \mathcal{L}\left(f, x_{0}, y_{0}, t_{k}, x_{0}\right)\right| \leq\left(\alpha_{1}(t) E+G\right)\left(L_{p}+M^{\prime}\right)+T\left|d\left(x_{0}\right)\right| \leq \beta_{1}\left(x_{0}\right), \\
& \quad\left|y_{1}^{p}\right| \leq\left|\overline{\mathcal{L}}\left(f^{p}, x_{0}^{p+1}, y_{0}^{p}, t, x_{0}\right)\right|+\left|d\left(x_{0}\right)\right|+\left|H \sum_{k=1}^{q} A_{k} \mathcal{L}\left(f^{p}, x_{0}^{p+1}, y_{0}^{p}, t_{k}, x_{0}\right)\right| \leq \\
& \left.\leq \mid \overline{\mathcal{L}}\left(f^{p}, x_{0}^{p+1}, y_{0}^{p}, t, x_{0}\right)-x_{0}^{p+1}, y_{0}^{p}, t_{k}, x_{0}\right)-\mathcal{L}\left(f, x_{0}, y_{0}, t, x_{0}\right)|+| \overline{\mathcal{L}}\left(f, x_{0}, y_{0}, t, x_{0}\right)|+| d\left(x_{0}\right) \mid+ \\
& \quad+\left|H \sum_{k=1}^{q} A_{k}\left[\mathcal{L}\left(f^{p}, x_{0}^{p+1}, y_{0}^{p}, t_{k}, x_{0}\right)-\mathcal{L}\left(f, x_{0}, y_{0}, t_{k}, x_{0}\right)\right]\right|+ \\
& +\left|H \sum_{k=1}^{q} A_{k} \mathcal{L}\left(f, x_{0}, y_{0}, t_{k}, x_{0}\right)\right| \leq 2\left(M+L_{p}\right)+G\left(M^{\prime}+L_{p}\right)+\left|d\left(x_{0}\right)\right| \leq \beta_{2}\left(x_{0}\right) .
\end{aligned}
$$

It follows that $x_{1}^{p+1}\left(t, x_{0}\right) \in D_{1}, \quad y_{1}^{p}\left(t, x_{0}\right) \in D_{2}$. By induction in a similar way we can establish that

$$
\left|x_{m}^{p+1}-x_{0}\right| \leq \beta_{1}\left(x_{0}\right), \quad\left|y_{m}^{p}\right| \leq \beta_{2}\left(x_{0}\right) .
$$


Now we consider the differences $x_{m}-x_{m}^{p+1}$ and $y_{m}-y_{m}^{p}$. For $m=1$ we have

$$
\begin{gathered}
\left|x_{1}-x_{1}^{p+1}\right| \leq\left|\mathcal{L}\left(f, x_{0}, y_{0}, t, x_{0}\right)-\mathcal{L}\left(f^{p}, x_{0}^{p+1}, y_{0}^{p}, t, x_{0}\right)\right|+ \\
+\left|T H \sum_{k=1}^{q} A_{k}\left[\mathcal{L}\left(f, x_{0}, y_{0}, t_{k}, x_{0}\right)-\mathcal{L}\left(f^{p}, x_{0}^{p+1}, y_{0}^{p}, t_{k}, x_{0}\right)\right]\right| \leq \\
\leq\left(\alpha_{1}(t) E+G\right) L_{p}, \\
\left|y_{1}-y_{1}^{p}\right| \leq\left|\overline{\mathcal{L}}\left(f, x_{0}, y_{0}, t, x_{0}\right)-\overline{\mathcal{L}}\left(f^{p}, x_{0}^{p+1}, y_{0}^{p}, t, x_{0}\right)\right|+ \\
+\left|H \sum_{k=1}^{q} A_{k}\left[\mathcal{L}\left(f, x_{0}, y_{0}, t_{k}, x_{0}\right)-\mathcal{L}\left(f^{p}, x_{0}^{p+1}, y_{0}^{p}, t_{k}, x_{0}\right)\right]\right| \leq \\
\leq\left(2 E+\frac{1}{T} G\right) L_{p} .
\end{gathered}
$$

Using (2.14)-(2.21) and Lemma 4 of [5] we get

$$
\begin{gathered}
\left|x_{2}-x_{2}^{p+1}\right| \leq\left|\mathcal{L}\left(f, x_{1}, y_{1}, t, x_{0}\right)-\mathcal{L}\left(f^{p}, x_{1}^{p+1}, y_{1}^{p}, t, x_{0}\right)\right|+ \\
+\left|T H \sum_{k=1}^{q} A_{k}\left[\mathcal{L}\left(f, x_{1}, y_{1}, t_{k}, x_{0}\right)-\mathcal{L}\left(f^{p}, x_{1}^{p+1}, y_{1}^{p}, t_{k}, x_{0}\right)\right]\right| \leq \\
\leq\left[\alpha_{1}(t) E+K_{1}\left(\alpha_{2}(t) E+\alpha_{1}(t) G\right)+\alpha_{1}(t) K_{2}\left(2 E+\frac{1}{T} G\right)\right] L_{p}+ \\
+\left|T H \sum_{k=1}^{q} A_{k}\left[\alpha_{1}\left(t_{k}\right) E+K_{1}\left(\alpha_{2}\left(t_{k}\right) E+\alpha_{1}\left(t_{k}\right) G\right)+\alpha_{1}\left(t_{k}\right) K_{2}\left(2 E+\frac{1}{T} G\right)\right] L_{p}\right| \leq \\
\leq\left(\alpha_{1}(t) E+G\right)\left[E+K_{1}\left(\frac{T}{3} E+G\right)+K_{2}\left(2 E+\frac{1}{T} G\right)\right] L_{p} \leq \\
\quad \leq\left(\alpha_{1}(t) E+G\right)[E+Q] L_{p}, \\
+\left|H \sum_{k=1}^{q} A_{k}\left[\mathcal{L}\left(f, x_{1}, y_{1}, t_{k}, x_{0}\right)-\mathcal{L}\left(f^{p}, x_{1}^{p+1}, y_{1}^{p}, t_{k}, x_{0}\right)\right]\right| \leq \\
\leq 2 \max _{t \in[0, T]}\left|f\left(t, x_{1}, y_{1}\right)-f^{p}\left(t, x_{1}^{p+1}, y_{1}^{p}\right)\right|+ \\
+\left|H \sum_{k=1}^{q} A_{k}\right| \mathcal{L}_{1}\left(f, x_{1}, y_{1}, t_{k}, x_{0}\right)-\mathcal{L}_{1}\left(f^{p}, x_{1}^{p+1}, y_{1}^{p}, t_{k}, x_{0}\right)|| \leq \\
\leq\left(2 E+\frac{1}{T} G\right)[E+Q] L_{p} .
\end{gathered}
$$

We can obtain by induction that

$$
\begin{aligned}
\left|x_{m}\left(t, x_{0}\right)-x_{m}^{p+1}\left(t, x_{0}\right)\right| & \leq\left(\alpha_{1}(t) E+G\right)\left[\sum_{i=1}^{m-1} Q^{i}\right] L_{p}, \\
\left|y_{m}\left(t, x_{0}\right)-y_{m}^{p}\left(t, x_{0}\right)\right| & \leq\left(2 E+\frac{1}{T} G\right)\left[\sum_{i=1}^{m-1} Q^{i}\right] L_{p} .
\end{aligned}
$$


Now we have to estimate $r_{m+1}\left(t, x_{0}\right)$ and $\widehat{r}_{m+1}\left(t, x_{0}\right)$ for every $m=0,1,2, \ldots$ by using Lemmas 3 and 4 of [5]:

$$
\begin{aligned}
& r_{1}\left(t, x_{0}\right) \leq\left|\mathcal{L}\left(f, x_{0}, y_{0}, t, x_{0}\right)\right|+T\left|d\left(x_{0}\right)\right|+ \\
& +\left|T H \sum_{k=1}^{q} A_{k} \mathcal{L}\left(f, x_{0}, y_{0}, t_{k}, x_{0}\right)\right| \leq\left(\frac{T}{2} E+G\right) M^{\prime}+T\left|d\left(x_{0}\right)\right| \equiv \gamma_{1}\left(x_{0}\right), \\
& \widehat{r}_{1}\left(t, x_{0}\right) \leq\left|\overline{\mathcal{L}}\left(f, x_{0}, y_{0}, t, x_{0}\right)\right|+\left|d\left(x_{0}\right)\right|+\left|H \sum_{k=1}^{q} A_{k} \mathcal{L}\left(f, x_{0}, y_{0}, t_{k}, x_{0}\right)\right| \leq \\
& \leq 2 M+\left|d\left(x_{0}\right)\right|+\frac{1}{T} G M^{\prime} \equiv \gamma_{2}\left(x_{0}\right), \\
& r_{2}\left(t, x_{0}\right) \leq\left|\mathcal{L}\left(f, x_{1}, y_{1}, t, x_{0}\right)-\mathcal{L}\left(f, x_{0}, y_{0}, t, x_{0}\right)\right|+ \\
& +\left|T H \sum_{k=1}^{q} A_{k}\left[\mathcal{L}\left(f, x_{1}, y_{1}, t_{k}, x_{0}\right)-\mathcal{L}\left(f, x_{0}, y_{0}, t_{k}, x_{0}\right)\right]\right| \leq \\
& \leq\left(1-\frac{t}{T}\right) \int_{0}^{t}\left[K_{1} r_{1}\left(\tau, x_{0}\right)+K_{2} \widehat{r}_{1}\left(\tau, x_{0}\right)\right] d \tau+\frac{t}{T} \int_{t}^{T}\left[K_{1} r_{1}\left(\tau, x_{0}\right)+K_{2} \widehat{r}_{1}\left(\tau, x_{0}\right)\right] d \tau+ \\
& +\mid T H \sum_{k=1}^{q} A_{k}\left[\left(1-\frac{t_{k}}{T}\right) \int_{0}^{t_{k}}\left[K_{1} r_{1}\left(\tau, x_{0}\right)+K_{2} \widehat{r}_{1}\left(\tau, x_{0}\right)\right] d \tau+\right. \\
& \left.+\frac{t_{k}}{T} \int_{t_{k}}^{T}\left[K_{1} r_{1}\left(\tau, x_{0}\right)+K_{2} \widehat{r}_{1}\left(\tau, x_{0}\right)\right] d \tau\right] \mid \leq\left(\alpha_{1}(t) E+G\right) \cdot\left[K_{1} \gamma_{1}\left(x_{0}\right)+K_{2} \gamma_{2}\left(x_{0}\right)\right] \text {, } \\
& \widehat{r}_{2}\left(t, x_{0}\right) \leq\left|\overline{\mathcal{L}}\left(f, x_{1}, y_{1}, t, x_{0}\right)-\overline{\mathcal{L}}\left(f, x_{0}, y_{0}, t, x_{0}\right)\right|+ \\
& +\left|T H \sum_{k=1}^{q} A_{k}\left[\mathcal{L}\left(f, x_{1}, y_{1}, t_{k}, x_{0}\right)-\mathcal{L}\left(f, x_{0}, y_{0}, t_{k}, x_{0}\right)\right]\right| \leq \\
& \leq 2 \max _{t \in[0, T]}\left|K_{1} r_{1}\left(\tau, x_{0}\right)+K_{2} \widehat{r}_{1}\left(\tau, x_{0}\right)\right|+ \\
& +\mid H \sum_{k=1}^{q} A_{k}\left[\left(1-\frac{t_{k}}{T}\right) \int_{0}^{t_{k}}\left[K_{1} r_{1}\left(\tau, x_{0}\right)+K_{2} \widehat{r}_{1}\left(\tau, x_{0}\right)\right] d \tau+\right. \\
& \left.+\frac{t_{k}}{T} \int_{t_{k}}^{T}\left[K_{1} r_{1}\left(\tau, x_{0}\right)+K_{2} \widehat{r}_{1}\left(\tau, x_{0}\right)\right] d \tau\right] \mid \leq\left(2 E+\frac{1}{T} G\right) \cdot\left[K_{1} \gamma_{1}\left(x_{0}\right)+K_{2} \gamma_{2}\left(x_{0}\right)\right] \text {. }
\end{aligned}
$$

Similarly, 


$$
\begin{gathered}
r_{3}\left(t, x_{0}\right) \leq\left\{\left(1-\frac{t}{T}\right) \int_{0}^{t}\left[K_{1}\left(\alpha_{1}(\tau) E+G\right)+K_{2}\left(2 E+\frac{1}{T} G\right)\right] d \tau+\right. \\
+\frac{t}{T} \int_{t}^{T}\left[K_{1}\left(\alpha_{1}(\tau) E+G\right)+K_{2}\left(2 E+\frac{1}{T} G\right)\right] d \tau+ \\
+\mid T H \sum_{k=1}^{q} A_{k}\left[\left(1-\frac{t_{k}}{T}\right) \int_{0}^{t_{k}}\left[K_{1}\left(\alpha_{1}(\tau) E+G\right)+K_{2}\left(2 E+\frac{1}{T} G\right)\right] d \tau+\right. \\
\left.\left.+\frac{t_{k}}{T} \int_{t_{k}}^{T}\left[K_{1}\left(\alpha_{1}(\tau) E+G\right)+K_{2}\left(2 E+\frac{1}{T} G\right)\right] d \tau\right] \mid\right\} \cdot\left[K_{1} \gamma_{1}\left(x_{0}\right)+K_{2} \gamma_{2}\left(x_{0}\right)\right] \leq \\
\leq\left(\alpha_{1}(t) E+G\right) \cdot Q \cdot\left[K_{1} \gamma_{1}\left(x_{0}\right)+K_{2} \gamma_{2}\left(x_{0}\right)\right], \\
+\mid H \sum_{k=1}^{q} A_{k}\left[\left(1-\frac{t_{k}}{T}\right) \int_{0}^{t_{k}}\left[K_{1}\left(\alpha_{1}(\tau) E+G\right)+K_{2}\left(2 E+\frac{1}{T} G\right)\right] d \tau+\right. \\
\left.\left.+\frac{t_{k}}{T} \int_{t_{k}}^{T}\left[K_{1}\left(\alpha_{1}(\tau) E+G\right)+K_{2}\left(2 E+\frac{1}{T} G\right)\right] d \tau\right] \mid\right\} \cdot\left[K_{1} \gamma_{1}\left(x_{0}\right)+K_{2} \gamma_{2}\left(x_{0}\right)\right] \leq \\
\leq\left(2 E+\frac{1}{T} G\right) \cdot Q \cdot\left[K_{1} \gamma_{1}\left(x_{0}\right)+K_{2} \gamma_{2}\left(x_{0}\right)\right] .
\end{gathered}
$$

We can show by induction that for arbitrary $m=0,1,2, \ldots$

$$
\begin{aligned}
& r_{m+1}\left(t, x_{0}\right) \leq\left(\alpha_{1}(t) E+G\right) \cdot Q^{m-1} \cdot\left[K_{1} \gamma_{1}\left(x_{0}\right)+K_{2} \gamma_{2}\left(x_{0}\right)\right], \\
& \widehat{r}_{m+1}\left(t, x_{0}\right) \leq\left(2 E+\frac{1}{T} G\right) \cdot Q^{m-1} \cdot\left[K_{1} \gamma_{1}\left(x_{0}\right)+K_{2} \gamma_{2}\left(x_{0}\right)\right] .
\end{aligned}
$$

From (2.24) and assumption c) we obtain the inequality

$$
\begin{gathered}
\left|x_{m+j}\left(t, x_{0}\right)-x_{m}\left(t, x_{0}\right)\right| \leq \sum_{i=0}^{j-1}\left|x_{m+i+1}\left(t, x_{0}\right)-x_{m+i}\left(t, x_{0}\right)\right| \leq \\
\leq \sum_{i=0}^{j-1} r_{m+i+1}\left(t, x_{0}\right) \leq \sum_{i=0}^{j-1}\left(\alpha_{1}(t) E+G\right) Q^{m+i-1}\left[K_{1} \gamma_{1}\left(x_{0}\right)+K_{2} \gamma_{2}\left(x_{0}\right)\right] \leq \\
\leq\left(\alpha_{1}(t) E+G\right) \cdot Q^{m-1}(E-Q)^{-1} \cdot\left[K_{1} \gamma_{1}\left(x_{0}\right)+K_{2} \gamma_{2}\left(x_{0}\right)\right] .
\end{gathered}
$$

For the derivatives $y_{m}\left(t, x_{0}\right)$ from $(2.25)$ in a similar way we have:

$$
\begin{gathered}
\left|y_{m+j}\left(t, x_{0}\right)-y_{m}\left(t, x_{0}\right)\right| \leq \\
\leq\left(2 E+\frac{1}{T} G\right) Q^{m-1}(E-Q)^{-1}\left[K_{1} \gamma_{1}\left(x_{0}\right)+K_{2} \gamma_{2}\left(x_{0}\right)\right] .
\end{gathered}
$$


It follows that (2.12) and (2.13) are uniformly convergent sequences:

$$
\lim _{m \rightarrow \infty} x_{m}\left(t, x_{0}\right)=x^{*}\left(t, x_{0}\right), \lim _{m \rightarrow \infty} y_{m}\left(t, x_{0}\right)=y^{*}\left(t, x_{0}\right) .
$$

Taking the limit as $j \rightarrow \infty$ in (2.26) and (2.27) we get the error estimates

$$
\begin{gathered}
\left|x^{*}\left(t, x_{0}\right)-x_{m}\left(t, x_{0}\right)\right| \leq\left(\alpha_{1}(t) E+G\right) \cdot Q^{m-1}(E-Q)^{-1} \cdot\left[K_{1} \gamma_{1}\left(x_{0}\right)+K_{2} \gamma_{2}\left(x_{0}\right)\right], \\
\left|y^{*}\left(t, x_{0}\right)-y_{m}\left(t, x_{0}\right)\right| \leq\left(2 E+\frac{1}{T} G\right) \cdot Q^{m-1}(E-Q)^{-1} \cdot\left[K_{1} \gamma_{1}\left(x_{0}\right)+K_{2} \gamma_{2}\left(x_{0}\right)\right] .
\end{gathered}
$$

Combining the last two inequalities with (2.22) and (2.23), we get the error estimates (2.10) and (2.11). Passing to the limit as $m \rightarrow \infty$ in (2.6) we obtain that $x^{*}\left(t, x_{0}\right)$ satisfies the integral equation

$$
x(t)=x_{0}+\mathcal{L}\left(f, x, y, t, x_{0}\right)+t H d\left(x_{0}\right)-t H \sum_{k=1}^{q} A_{k} \mathcal{L}\left(f, x, y, t_{k}, x_{0}\right) .
$$

While differentiating it, we get that $x^{*}\left(t, x_{0}\right)$ is a solution of the perturbed BVP (2.8)-(2.9).

The following statement gives necessary and sufficient conditions for the existence of a solution of the BVP (2.1)-(2.2).

Theorem 2. Under the conditions of Theorem 1, the limit function $x^{*}\left(t, x_{0}^{*}\right)$ is a solution of the BVP (2.1)-(2.2) if and only if $x_{0}^{*}$ verifies the determining equation

$$
\begin{aligned}
\Delta\left(x_{0}\right)=- & \frac{1}{T} \int_{0}^{T} f\left(s, x^{*}\left(s, x_{0}\right), y^{*}\left(s, x_{0}\right)\right) d s+H d\left(x_{0}\right)+ \\
& +H \sum_{k=1}^{q} A_{k} \mathcal{L}\left(f, x^{*}, y^{*}, t_{k}, x_{0}\right)=0 .
\end{aligned}
$$

Proof. The proof can be carried out in the same way as for the corresponding statements from [2] (Theorem 2.3).

\section{Sufficient existence conditions}

Consider the $m$-th approximation to the determining equation (2.28)

$$
\begin{aligned}
\Delta_{m}^{p}\left(x_{0}\right)= & -\frac{1}{T} \int_{0}^{T} f^{p}\left(s, x_{m}^{p+1}\left(s, x_{0}\right), y_{m}^{p}\left(s, x_{0}\right)\right) d s+H d\left(x_{0}\right)+ \\
& +H \sum_{k=1}^{q} A_{k} \mathcal{L}\left(f^{p}, x_{m}^{p+1}, y_{m}^{p}, t_{k}, x_{0}\right)=0 .
\end{aligned}
$$

Theorem 3. Suppose that the conditions of Theorem 1 hold. Furthermore, assume that

d) there exists a closed, convex subset $D^{\prime}=D_{1}^{\prime} \times D_{2}^{\prime} \subset D_{1} \times D_{2}$ so that for arbitrary $m$ and fixed $p$ the approximate determining equation (3.1) has only one solution $x_{0}=x_{0 m}^{p}$ with non-zero topological index; 
e) on the boundary $\partial D$ of the subset $D$ the inequality

$$
\inf _{x_{0} \in \partial D}\left|\Delta_{m}^{p}\left(x_{0}\right)\right|>\left(E+\frac{1}{T} G\right) W_{m}^{p}
$$

holds.

Then there exists a solution $x=x^{*}(t)$ to the BVP (2.1)-2.2) with the initial value $x^{*}(0)=x_{0}^{*}$, where $x_{0}^{*} \in D_{1}^{\prime}$.

Proof. Similarly to (2.15) and making use of (2.10) and (2.11), we get

$\left|f\left(t, x^{*}, y^{*}\right)-f^{p}\left(t, x_{m}^{p+1}, y_{m}^{p}\right)\right| \leq\left[K_{1}\left(\alpha_{1}(t) E+G\right)+K_{2}\left(2 E+\frac{1}{T} G\right)\right] W_{m-1}^{p}+L_{p}$.

For the deviation of the exact and approximate determining functions we have that

$$
\begin{gathered}
\left|\Delta\left(x_{0}\right)-\Delta_{m}^{p}\left(x_{0}\right)\right| \leq \frac{1}{T} \int_{0}^{T} \mid f^{p}\left(s, x^{*}\left(s, x_{0}\right), y^{*}\left(s, x_{0}\right)\right)- \\
-f^{p}\left(s, x_{m}^{p+1}\left(s, x_{0}\right), y_{m}^{p}\left(s, x_{0}\right)\right)\left|+H \sum_{k=1}^{q} A_{k}\right| \mathcal{L}\left(f^{p}, x^{*}, y^{*}, t_{k}, x_{0}\right)- \\
-\mathcal{L}\left(f^{p}, x_{m}^{p+1}, y_{m}^{p}, t_{k}, x_{0}\right) \mid \leq\left(E+\frac{1}{T} G\right)\left(Q W_{m-1}^{p}+L_{p}\right) \leq\left(E+\frac{1}{T} G\right) W_{m}^{p} .
\end{gathered}
$$

Similarly to Theorem 3.1 of [2], one can prove that the vector fields $\Delta\left(x_{0}\right)$ and $\Delta_{m}^{p}\left(x_{0}\right)$ are homotopic, which completes the proof of Theorem 3.

\section{REFERENCES}

[1] Samoilenko, A. M. and Rontó, N. I.: Numerical-Analytic Methods of Investigating Solutions of Boundary Value Problems, Naukova Dumka, Kiev, 1985 (in Russian).

[2] Samoilenko, A. M. and Rontó, N. I.: Numerical-Analytic Methods in the Theory of Boundary Value Problems, Naukova Dumka, Kiev, 1992 (in Russian).

[3] Rontó, M. and Samoilenko, A. M.: Numerical-Analytic Methods in the Theory of Boundary Value Problems, World Scientific, Singapore, 2000.

[4] Korol, I. I. and Korol, I. YU.: Using of polynomial approximation method for solving of multi-point BVPs, Naukovij Visnik Uzhgorods'koho Universitetu, Matematika, 4, (1999), 71-78.

[5] Rontó, M. and MészÁros, J.: Some remarks on the convergence analysis of the numerical-analytic method based upon successive approximations, Ukrainskij Matematicheskij Zhurnal, 48(1), (1996), 90-95. 